\title{
Mikrobieller Plastikabbau im Meer: die Suche nach dem Unwahrscheinlichen
}

\author{
SONJA OBERBECKMANN ${ }^{1}$, STEPHANIE MARKERT ${ }^{2,3}$, MATTHIAS LABRENZ ${ }^{1}$ \\ ${ }^{1}$ SEKTION BIOLOGISCHE MEERESKUNDE, LEIBNIZ-INSTITUT FÜR OSTSEEFORSCHUNG \\ WARNEMÜNDE (IOW), ROSTOCK \\ 2 INSTITUT FÜR PHARMAZIE, UNIVERSITÄT GREIFSWALD \\ ${ }^{3}$ INSTITUT FÜR MARINE BIOTECHNOLOGIE, GREIFSWALD
}

Microplastic particles have been found in all the world's oceans. Since their surfaces are microbially colonised, research is currently determining whether microbes are able to degrade the synthetic polymers. We show that their contribution to the biodegradation of plastics seems to be extremely limited. Also, it is unlikely that microbes specialise in plastic degradation through evolutionary adaptation. This is not only due to the great diversity and simultaneous very low bioavailability of microplastics in the oceans, but also because it is energetically disadvantageous.

DOI: $10.1007 / \mathrm{s} 12268-021-1591-7$

(C) Die Autoren 2021

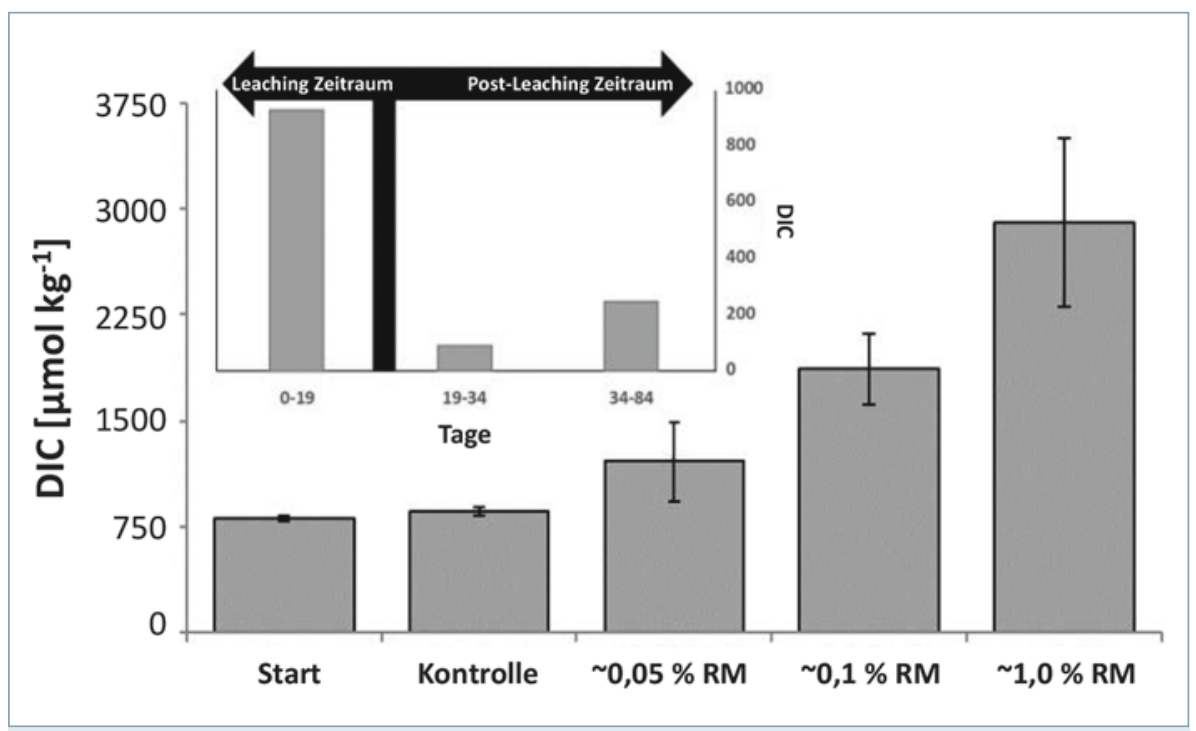

$\Delta$ Abb. 1: Polyamid-6-Partikel (PA6) mit unterschiedlichem Gehalt an Restmonomeren (RM) wurden einer mikroorganismenhaltigen Wasserprobe ausgesetzt und die Entstehung von gelöstem anorganischem Kohlenstoff (DIC; primär zusammengesetzt aus $\mathrm{CO}_{2}, \mathrm{HCO}_{3}{ }^{-}$und $\mathrm{CO}_{3}{ }^{2-}$ ) gemessen. Mit zunehmendem RM-Gehalt im Polymer erhöhte sich auch der DIC-Anteil. Die DIC-Bildung resultiert daher offenbar nicht aus mikrobiellem Abbau des Polymers PA6, sondern lediglich aus der Zersetzung der Restmonomere. Zum Vergleich sind der Ausgangs-DIC-Anteil vor Beginn (Start) sowie die Kontrolle ohne PA6 zu sehen. Einschub oben links: zeitliche Entwicklung des DIC-Gehalts bei Inkubation von PA6 mit 0,1\% RM. Während der ersten 19 Tage wurde deutlich mehr DIC-Bildung gemessen (Leaching-Zeitraum) als anschließend, was den Schluss nahelegt, dass nach der mikrobiellen Zersetzung aller verfügbaren RM keine weitere Biodegradation stattfindet (modifiziert nach [6]).
Kunststoff ist mittlerweile allgegenwärtig in aquatischen Ökosystemen. Dort zerfallen die wasserunlöslichen größeren Kunststoffe häufig durch UV-Strahlung, mechanischen Abrieb und Auswaschung von Weichmachern in Partikel kleiner als fünf Millimeter. Auf diesem Mikroplastik reichern sich schnell Mikroorganismen an, da von Wasser umgebene Oberflächen generell Hotspots für mikrobielle Besiedelung sind [1, 2]. Gründe dafür sind höhere Nährstoffkonzentrationen als im umgebenden Wasser, Schutz vor ungünstigen Umweltbedingungen und der enge Austausch mit anderen Biofilmmitgliedern. Mikrobielle Gemeinschaften auf Mikroplastik unterscheiden sich in der Regel von freilebenden Gemeinschaften des umgebenden Wassers, weisen aber starke Gemeinsamkeiten zu Biofilmen auf anderen Oberflächen, wie Holz, Glas oder Cellulose, auf [3, 4]. Einzelne Gruppen, beispielsweise Vertreter der Rhodobacteraceae und Sphingomonadaceae, scheinen allerdings eine besondere Rolle in Mikroplastikbiofilmen zu spielen [3].

Zurzeit ist unklar, ob die Organismen in mikrobiellen Biofilmen das Mikroplastik auch abbauen können. Interdisziplinär unterschiedliche Auslegungen des Begriffes „bioabbaubar“ und damit verbundene verschiedene Interpretationen von Abbauexperimenten in situ und in vitro sowie die begrenzte Übertragbarkeit von Ergebnissen verstärken diese Unklarheiten noch. In einem Übersichtsartikel haben wir die Problematik wie folgt zusammengefasst und definiert [3].

\section{Definition von Biodegradation}

Die Biodegradation von Plastik kann in drei Abschnitte unterteilt werden [5]: Verwitterung, Fragmentierung und Mineralisation. Zuerst erweitern Verwitterung und Fragmentierung die Polymeroberfläche und reduzieren dessen Molekulargewicht, was die nachfolgende potenzielle mikrobielle Spaltung ermöglicht. Am Ende steht der Abbau im biologischen Sinne, also die vollständige Mineralisierung in $\mathrm{CO}_{2}, \mathrm{H}_{2} \mathrm{O}$ und Salze, begleitet von der Bildung neuer Biomasse. 
Anders bewerten Polymerwissenschaftler oder die Kunststoffindustrie den Abbau: Ihnen reicht die Fragmentierung des Kunststoffs zu Mikroplastik bei gesteuerten Abbauprozessen, z. B. der Kompostierung, meist aus, um Abbaubarkeit zu postulieren. Diese unterschiedlichen Interpretationen sind für die Öffentlichkeit nicht einfach zu durchschauen. Für uns und den weiteren Verlauf dieses Artikels beschreibt „biologisch abbaubar" die vollständige Mineralisierung von Kunststoffen.

\section{Zersetzung von Kunststoffen - im Labor anders als im Ozean}

Ergebnisse aus definierten Laborexperimenten können häufig nur eingeschränkt auf die Umwelt mit ihren ganz anderen Bedingungen übertragen werden. Dies gilt auch für den mikrobiellen Kunststoffabbau. So sind synthetische Polymere energiereich und stellen für Mikroorganismen theoretisch eine gute Energie- und Kohlenstoffquelle dar, prinzipiell vergleichbar mit dem gesättigten Kohlenwasserstoff Oktadekan $\left(\mathrm{C}_{18} \mathrm{H}_{38}\right)$. Häufig sind aber selbst unter Laborbedingungen Abbauexperimente mit herkömmlichen und im Meer häufig zu findenden Kunststoffen, wie Plastikflaschen oder -tüten, schwer zu interpretieren. Wir führten daher eine Abbaustudie mit additivfreiem Polyamidmikroplastik durch [6]. Es wurden unterschiedliche Volumen/Oberflächen-Verhältnisse angeboten, was aufgrund unterschiedlicher mikrobieller Angriffsmöglichkeiten prinzipiell zu unterschiedlichen Abbauraten führen sollte. Tatsächlich verzeichneten wir die Bildung von $\mathrm{CO}_{2}$ mit einem deutlich geringeren Anteil von ${ }^{14} \mathrm{C}$. Da synthetische Polymere aus Petrochemikalien hergestellt werden, in denen aufgrund des radioaktiven Zerfalls kein ${ }^{14} \mathrm{C}$ enthalten ist, weist dies eindeutig auf den Plastikursprung des $\mathrm{CO}_{2}$ hin. Die $\mathrm{CO}_{2}$-Gehalte unterschieden sich aber nicht signifikant zwischen den Proben, wie man es bei einem Abbau der unterschiedlich großen Mikroplastikfraktionen erwartet hätte. Vielmehr wiesen wir die Mineralisation unpolymerisierter Monomere des untersuchten Polyamids nach: Der Abbau eines Polymers war also nur vorgetäuscht (Abb. 1).

Plastikadditive und Monomerreste sind weit verbreitete Bestandteile von Plastikprodukten. Da sie aber häufig nicht benannt sind, bilden sowohl Plastikprodukte des täglichen Lebens als auch industrielle Standardplastikpellets eine schlechte Grundlage für

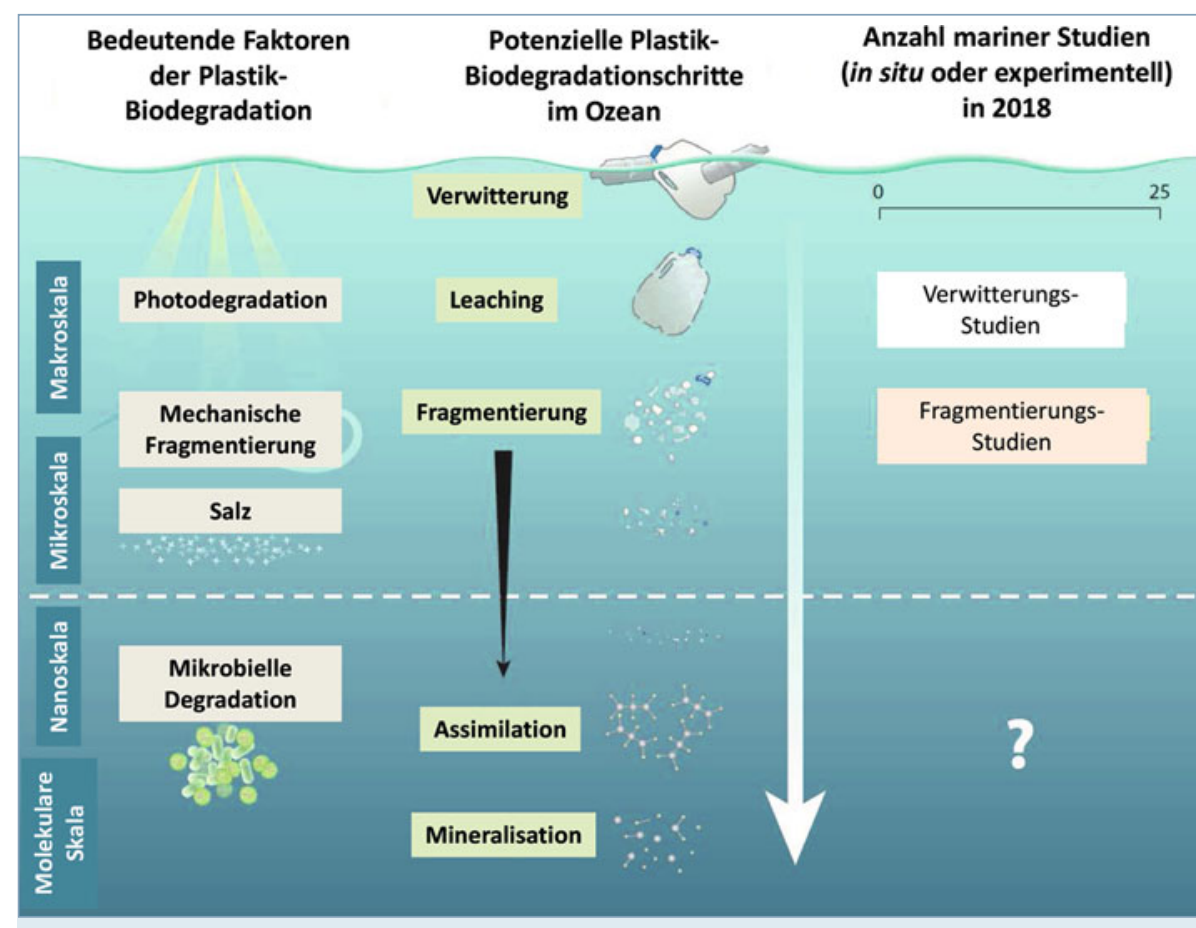

$\Delta$ Abb. 2: Wesentliche Umweltfaktoren, die den Abbau von Kunststoff im Ozean bis hin zu noch sichtbarem Mikroplastik beeinflussen. Insbesondere physikalisch-chemische Kräfte führen zum ersten Schritt des Plastikabbaus: Verwitterung, Leaching und Fragmentierung makroskopischer Plastikteile. Diese Faktoren wirken aber kaum in noch kleineren Größenbereichen bis hin zur molekularen Skala. Weitere Plastikzersetzung müsste dort primär mikrobieller Assimilation und

Mineralisation unterliegen. Diese Abbauschritte (unterhalb der horizontalen gestrichelten Linie) können teilweise aus optimierten Laborexperimenten extrapoliert werden (links vom Pfeil), wurden aber für den Ozean bis jetzt noch nie in Studien belegt (rechts vom Pfeil) (modifiziert nach [3]).

Abbauexperimente, da der Polymerabbau leicht überschätzt werden kann.

Prominentes Beispiel für einen vollständigen Abbau von Polyethylenterephthalat (PET) ist das Bakterium Iodanella sakaiensis, das aus einer PET-kontaminierten Probe außerhalb einer PET-Recyclingeinrichtung isoliert wurde [7]. I. sakaiensis kann PET unter optimalen Laborbedingungen mithilfe von zwei Enzymen vollständig mineralisieren und ausschließlich auf der Energiequelle PET wachsen. Ist im Labor die vollständige Mineralisierung von synthetischen Polymeren bis hin zum $\mathrm{CO}_{2}$ methodisch prinzipiell nachweisbar, insbesondere für weniger stabile Plastikpolymere wie PET, stellt sich dies für den Ozean allerdings ganz anders dar.

Um hier potenziellen Plastikabbau verfolgen zu können, inkubieren Kunststoffabbauexperimente im Ozean z. T. über Wochen bis Jahre, sodass allein aus methodischen Gründen potenzieller biologischer Abbau von Mikrokunststoffen bisher nur auf der Grundlage des Gewichtsverlusts abgeleitet wurde. Der Gewichtsverlust allein, selbst in Kombination mit mikrobiellem Wachstum, erlaubt aber keine Unterscheidung zwischen einem
Abbau der Polymere oder schlichter Fragmentierung bzw. Additivverlust. Wir durchsuchten die Plattform Web of Science systematisch nach Publikationen zur Biodegradation von Mikroplastik im Ozean, wurden aber nicht fündig. Bis heute beschränken sich entsprechende Studien allein auf die Verwitterung und Fragmentierung von Mikroplastik (Abb. 2, [3]).

\section{Plastikoberflächen spielen für Meeresbakterien keine spezifische Rolle}

Wie übertragbar sind im Labor ermittelte Degradationsraten auf den Ozean? Können Mikroorganismen in marinen Habitaten überhaupt einen Vorteil aus einem vollständigen Abbau von Mikroplastik ziehen? Aus Martin Alexanders „Biodegradations-Dekalog“ [8] lässt sich ableiten, dass in der marinen Umwelt nicht der Energiegehalt der synthetischen Polymere die Abbaubarkeit bestimmt. Vielmehr sind es Faktoren wie die extrem niedrige Bioverfügbarkeit, eine sehr hohe chemische Stabilität und die Tatsache, dass die große Vielfalt an Kunststoffpolymeren eine ebenso große Bandbreite an Abbau- 


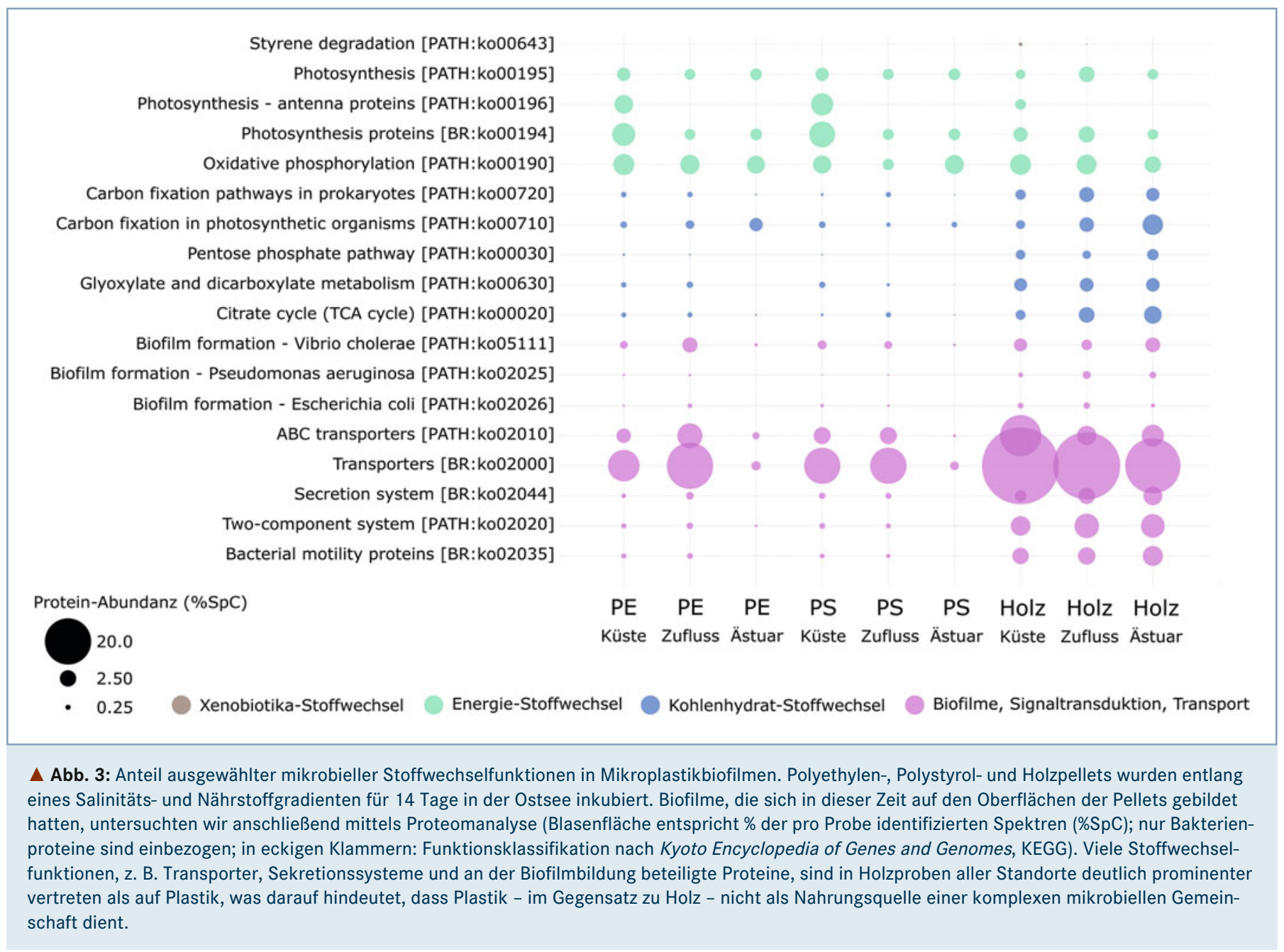

wegen erfordern würde [3]. Diese Faktoren lassen die These zu, dass Mikroplastikabbau im Ozean für Mikroorganismen energetisch unvorteilhaft ist und daher nicht aktiv stattfindet.

Bisher existieren nur vereinzelte metagenomische Studien von Mikroplastikbiofilmen im Ozean, aber diese scheinen die These zu bestätigen. Mutmaßlich am Xenibiotikaabbau beteiligte Gene finden sich häufiger auf Plastik als im Umgebungswasser [9], was aber auch auf den Abbau plastikassoziierter Schadstoffe zurückzuführen sein könnte. Wahrscheinlich nutzen mikrobielle Plastikbesiedler in erster Linie Kohlenstoff und andere Nährstoffe, die von filtrierenden Bryozoa, anderen marinen Eukaryoten und autotropher Aktivität der Biofilmgemeinschaft angesammelt werden [9]. Die Auswertung von 108 marinen und 25 terrestrischen Metagenomen in einer Metastudie zur globalen Verteilung von PET-abbauenden Enzymen [10] ergab, dass PET-Abbau in marinen Habitaten keine Rolle spielt. In den Datensätzen kamen PET-Hydrolasen kaum vor, am häufig- sten wurden sie in der Nähe einer Schweröllagerstätte gefunden.

In einer eigenen Studie führten wir erstmalig eine kombinierte Metagenom- und Metaproteomanalyse durch, um die Lebensstile und potenzielle mikrobielle Plastikabbauprozesse der Biofilmbewohner auf Mikroplastik in situ näher zu beleuchten [11]. Dafür inkubierten wir entlang eines Salinitäts- und Nährstoffgradienten in der Ostsee Polyethylen-, Polystyrol- und Holzpellets. Detektierte Gene und Proteine deuten auf einen auffallend materialunspezifischen Metabolismus auf Mikroplastik hin, wohingegen Holz im selben Zeitraum die Bildung von substratspezifischen Biofilmen induziert und selbst als Nährstoffquelle dient (Abb. 3). Auf Polyethylen und Polystyrol waren solche Proteine signifikant häufiger als auf Holz, die an der Cytoskelettstruktur eukaryotischer Zellen beteiligt sind oder unspezifische, das normale Wachstum begleitende (vegetative) Funktionen haben. Auf Holz waren spezifische, substratabbaubezogene Funktionen (z. B. Beta-Mannanase, Beta-1,4-xylanase,
Beta-Xylosidase) deutlich häufiger. Unsere Studie weist darauf hin, dass Kunststoffe in marinen Habitaten eher einer opportunistischen Besiedlung, z. B. durch phototrophe Bakterien, unterliegen und weniger einem biologischen Abbau.

\section{Mikroben können Plastikproblem des Ozeans nicht lösen}

Ein biologischer Abbau von Mikroplastik konnte in der Meeresumwelt bisher nicht nachgewiesen werden. Aufgrund der geringen Bioverfügbarkeit von Kunststoffen wird deren Abbau hauptsächlich durch physikalisch-chemische Kräfte bestimmt und endet nach heutigem Kenntnisstand mit der Anreicherung von Mikroplastik und Nanoplastik im marinen System. Daher ist eine „biologische“ Reduzierung des Plastikmülls in den Weltmeeren durch mikrobiellen Abbau unwahrscheinlich. Allein die gezielte Vermeidung von Plastikeintrag in die Ozeane durch effiziente - auch proaktive - Maßnahmen kann dieses globale Problem eindämmen. 


\section{Literatur}

[1] Azam F (1998) Microbial control of oceanic carbon flux: The plot thickens. Science 280: 694-696

[2] Zobell C (1943) The effect of solid surfaces upon bacterial activity. J Bacteriol 46: 39-56

[3] Oberbeckamm S, Labrenz M (2020) Marine microbia assemblages on microplastics: diversity, adaptation, and role in degradation. Annu Rev Mar Sci 12: 209-232

[4] Wright RJ, Erni-Cassola G, Zadjelovic V et al. (2020)

Marine plastic debris: a new surface for microbial colonization. Environ Sci Technol 54: 11657-11672

[5] Andrady AL (2017) The plastic in microplastics: A review. Mar Poll Bull 119: 12-22

[6] Klaeger F, Tagg F, Tagg AS et al. (2019) Residual monomer content afects the Interpretation of plastic degradation. Sci Rep 9: 2120

[7] Yoshida S, Hirada K, Takehana T et al. (2016) A bacterium that degrades and assimilates poly(ethylene terephthalate). Science 351: 1196-1199

[8] Alexander M (1975) Environmental and microbiological problems arising from recalcitrant molecules. Microb Ecol 2: $17-27$
[9] Bryant JA, Clemente TM, Viviani DA et al. (2016) Diversity and activity of communities inhabiting plastic debris in the North Pacific Gyre. mSystems 1: e00024-16 [10] Danso D, Schmeisser C, Chow J et al. (2018) New insights into the function and global distribution of polyethyene terephthalate (PET)-degrading bacteria and enzymes in marine and terrestrial metagenomes. Appl Environ Microbio 84: $\mathrm{e} 02773-17$

[11] Oberbeckmann S, Bartosik D, Werner J et al. (2021) Genomic and proteomic profiles of biofilms on microplastics are decoupled from artificial surface properties. Env Microbiol, https://doi.org/10.1111/1462-2920.15531

Funding note: Open Access funding enabled and organized by Projekt DEAL. Open Access: Dieser Artikel wird unter der Creative Commons Namensnennu 4.0 International Lizenz veroffentlicht, welche die Nutzung, Vervielfältigung, Bearbeitung, Verbreitung und Wiedergabe in jeglichem Medium und Forma

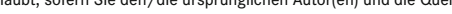

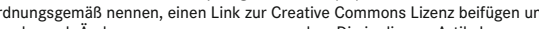
enthaltenen Bilder und sonstiges Drittmoterial unterlin diesem Artikel genannten Creative Commons Lizenz, sofern sich aus der Abbildungslegende nichts anderes ergibt. Sofern das betreffende Material nicht unter der genannten Creative Commons Lizenz steht und die betreffende Handlung nich Weiterverwendungen des Materials die Einwilligung des jeweiligen Rechteinhabers einzuholen. Weitere Details zur Lizenz entnehmen Sie bitte der Lizenzinformation auf http://creativecommons.org/licenses/by/4.0/deed.de.

Korrespondenzadresse:

Prof. Dr. Matthias Labrenz

Umweltmikrobiologie

Leibniz-Institut für Ostseeforschung

Warnemünde - IOW

Seestraße 15

D-18119 Rostock-Warnemünde

matthias.labrenz@io-warnemuende.de www.io-warnemuende.de/environmental-micro biology.html

\section{AUTOREN}

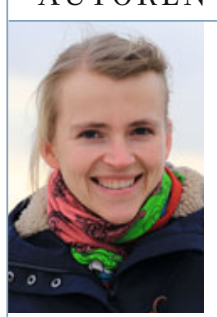

Sonja Oberbeckmann 2001-2007 Biologiestudium an der Universität Göttingen. 2007-2011 Promotion am Alfred-Wegener-Institut, Helmholtz-Zentrum für Polar- und Meeresforschung, Standort Helgoland. 2011-2013 Postdoc an den Universitäten Hull und Lincoln, UK. 2013-2020 Postdoc, Wissenschaftliche Mitarbeiterin und Projektleiterin am LeibnizInstitut für Ostseeforschung Warnemünde; dort seit 2020 Habilitationsstelle.

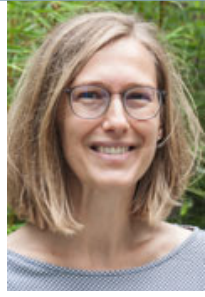

\section{Stephanie Markert}

1998-2003 Biologiestudium an der Universität Greifswald. 2003-2008 Promotion am Institut für Mikrobiologie der Universität Greifswald bei Prof. Dr. M. Hecker. Seit 2008 Wissenschaftliche Mitarbeiterin und Projektleiterin am Institut für Pharmazie der Universität Greifswald und am Institut für Marine Biotechnologie e. V. mit den Schwerpunkten Marine Symbiosen und Marine Metaproteomics.

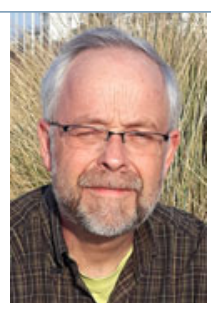

Matthias Labrenz 1995-2003 Promotion an der Universität Kiel und Postdoc an der University of Wisconsin-Madison, USA 2003 Wissenschaftlicher Mitarbeiter, Leibniz-Institut für Ostseeforschung Warnemünde (IOW), Rostock. 2008 Habilitation im Fach Mikrobiologie, Universität Rostock. 2012 Gruppenleiter Umweltmikrobiologie, IOW. 2018 Associate Professor für Mikrobielle Ökologie, Universität Klaipeda, Litauen. Seit 2019 Professor für Umweltmikrobiologie, Universität Rostock. 